\title{
Supermassive Black Holes, the Early Universe, and Gamma-Ray Bursts
}

\author{
Shawqi Al Dallal ${ }^{1}$, Walid J. Azzam ${ }^{2}$ \\ ${ }^{1}$ College of Graduate Studies and Research, Ahlia University, Bahrain \\ ${ }^{2}$ Department of Physics, College of Science, University of Bahrain, Bahrain \\ Email:wjazzam@gmail.com
}

Received April 18, 2013; revised May 25, 2013; accepted June 27, 2013

Copyright (C) 2013 Shawqi Al Dallal, Walid J. Azzam. This is an open access article distributed under the Creative Commons Attribution License, which permits unrestricted use, distribution, and reproduction in any medium, provided the original work is properly cited.

\begin{abstract}
Observational evidence reveals that supermassive black holes reside at the center of most galaxies up to the furthest observable redshifts. The tight $\mathrm{M}-\sigma$ relation suggests a close operative feedback between the growth of supermassive black holes and the growth of the galactic bulge. Models describing the formation scenarios of seeding black holes and their growth are reviewed. In each of these models, the prevailing environments in the primordial-galactic disks, including the gas dynamics, cooling processes, and metallic enrichment are explored. It is shown that the galactic disk parameters set constraints on the channel of formation of the seeding black holes and their growth. Primordial black holes from the inflationary era, their formation, possible interaction, and constraints on their observations are discussed. Gamma-ray bursts resulting either from the collapse of massive stars, or from the collision of compact objects are explored. The abundance of these violent events in the early universe suggests a possible connection with galaxy formation.
\end{abstract}

Keywords: Black Holes; Early Universe; Gamma-Ray Bursts

\section{Introduction}

Astronomical observations and theoretical studies during the past few decades have revealed the existence of black holes with a wide spectrum of masses in the early universe [1-3]. However, current observations remain incapable of directly probing the formation of supermassive black holes, despite their importance in setting the stage for the subsequent evolution of galaxies [4-6]. The demography of black holes at the center of galaxies is an important feature and a promising channel to enhance our understanding of galaxy formation [7]. The Hubble Space Telescope and the Chandra X-ray Observatory detected supermassive black holes with masses in excess of one billion $M_{\odot}$ in quasars at redshifts that correspond to just few hundred million years after the Big Bang [8,9]. The existence of supermassive black holes imposes important constraints on their formation mechanism [10]. Furthermore, the gas physics involved in their formation is yet to be understood $[5,11]$. There is no decisive answer concerning the formation of the earliest black holes, primarily because their growth process masks the origin and properties of the initial progenitor. The logical question that might arise is to what extent modern astronomical observatories can set the stage for probing the evolutionary stages in galactic formation. An answer to this question may be provided by future observatories such as the James Webb Telescope.

In this paper we review some theoretical and observational studies of black holes in the early universe, their origin, formation, and fate. In the first part, we outline the observational evidence concerning the existence of supermassive black holes in the early universe, followed by a brief introduction to the techniques employed in the determination of their masses. In the second part, we present the various models for the formation of black holes in the early universe, including the collapse of population III stars, dynamic instabilities, collapse of gas due dynamic instabilities, the collapse of supermassive stars, the dynamical processes in enriched halos that lead to the formation of massive black holes, and the formation and fate of primordial black holes. The last section of this paper is devoted to the connection between black holes and gamma-ray bursts. 


\section{Observational Evidence of Supermassive Black Holes}

Astronomical observations reveal that supermassive black holes lurk at the centers of galaxies all the way up to the furthest observable redshifts [12]. The first discovered supermassive black hole is the one at the center of the Milky Way Galaxy, which coincides with the location of Sagittarius $A$. The Milky Way black hole has an estimated mass of 4.1 million solar masses $\left(M_{\odot}\right)$ [13], which is relatively small when compared to the 230 million $M_{\odot}$ black hole at the center of the Andromeda Galaxy [14], or the 6.4 billion $M_{\odot}$ supermassive black hole lurking at the center of M87 [15,16]. The most massive supermassive black hole discovered so far has a mass of 21 billion $M_{\odot}$ and resides at the center of the NGC 4889 galaxy in the Coma cluster [17]. Galaxy mergers may give rise to binary supermassive black holes as in the case of the binary system OJ287 [18]. An interesting property of supermassive black holes is their relatively low density, which is defined as the black hole's mass divided by its Schwarzschild volume. It can easily be shown that the density of a supermassive black hole is inversely proportional to the square of its mass, and accordingly, since it has very high mass, its density is usually low.

The formation of supermassive black holes in the early universe remains one of the most controversial subjects in astrophysics. The main issue is that our current observational tools are incapable of effectively differentiating between the predictions of various models. Many techniques have been developed to probe this issue. The Chandra Deep Field South (CDFS), combined with very deep optical and infrared images from the Hubble Space Telescope gave astronomers the opportunity to look for black holes lurking in about 200 galaxies at an era when the universe was between 800 million to 950 million years old [19]. The observations reveal that between $30 \%$ and $100 \%$ of distant galaxies contain growing supermassive black holes. When these results are extrapolated to the full sky, we arrive at the huge number of about 30 million supermassive black holes in the early universe $[20,21]$. One of the main objectives in studying supermassive black holes is to understand their formation and growth. From an observational perspective, optical telescopes are not the right tool to use since they are unable to penetrate the thick cloud of gas and dust that enshrouds nearly all black holes. Only high energy X-rays can find their way through the thick veil of gas and dust which makes the Chandra X-Ray Observatory the right tool for achieving this task.

\section{Estimation of the Mass of Supermassive Black Holes}

The determination of the masses of black holes is an es- sential element in verifying the extent of accuracy of various galactic formation and black hole growth models and their adherence to observations. Two important techniques are employed to estimate the masses of black holes at the centers of galactic bulges. The first technique, known as reverberation mapping, is the primary approach in which the black hole mass is determined directly from observational data, though in certain cases the estimation of some parameters concerning gas dynamics is needed. The second approach depends on establishing a correlation between the masses of black holes and certain parameters of the hosting bulges. Both techniques will be briefly outlined below.

\subsection{Reverberation Mapping}

Reverberation mapping is the primary mass estimation technique for determining the masses of supermassive black holes at the centers of galaxies. The technique involves measuring the structure of the broad emission line region (BLR) around a supermassive black hole, where the mass is measured directly from the gravitationally induced motion of the nearby gas [22]. The equation regulating this process is given by

$$
G M=f R_{B L R}(\Delta V)^{2}
$$

where $G$ is the gravitational constant, $\Delta V$ is the rms velocity of gas moving near the black hole as measured from the Doppler broadening of the gaseous emission lines, $R_{B L R}$ is the radius of the broad-line region, and $f$ is a form factor that depends on the shape of the BLR. The measurement of $R_{B L R}$ is considered a serious challenge. To perform the measurement, the adopted standard technique is based on the fact that the emission line fluxes experience strong variation in response to changes in the continuum, determined by the light from the accretion disk near the black hole [23]. Moreover, these lines have a certain delay with respect to changes in the continuum, presumably due to the light travel time, which permits the measurement of $R_{B L R}$. Measurement of the $f$ factor presents an added difficulty. Simple models of BLR were used until about 2004 to estimate $f$. More recently, $f$ has been determined by bringing the $\mathrm{M}$-sigma relation for active and quiescent galaxies into agreement [22].

\subsection{The M-Sigma Relation}

The correlation between the masses of supermassive black holes and the velocity dispersion of their host bulge was first demonstrated by Merritt [24] and Ferrarese and Merritt [25]. This relation is used to estimate the black holes masses in far away galaxies where no direct mass measurement can be made, and is given by

$$
\frac{M}{10^{8} M_{\odot}} \approx A\left[\frac{\sigma}{200 \mathrm{~km} / \mathrm{s}}\right]^{\alpha}
$$


where $A$ is a constant of order $3, \sigma$ is the stellar velocity dispersion of the galaxy bulge, and $\alpha$ is a constant of order 5 representing the slope of the $M-\sigma$ relation. Ferrarese and Merritt [25] found $A=3.1$ and $\alpha=4.8 \pm 0.5$. To establish the above relation, the above authors assumed a central velocity dispersion, $\sigma_{c}$, that was corrected for an effective aperture of radius $r_{e} / 8$, where $r_{e}$ is the half-light radius. Gebhardt et al. [26] used, as an independent variable, the dispersion $\sigma_{e}$ which is defined as the spatially averaged rms line-of-sight stellar velocity within the effective radius $r_{e}$. They found a smaller slope value: $\alpha=3.75 \pm 0.3$ and a greater vertical scatter. Merritt and Ferrarese [27] combined the stellar gas dynamics and the reverberation mapping mass estimates to derive a best fit relation with $A=1.3 \pm 0.36$, and $\alpha=4.72 \pm 0.36$. King [28] relied on observations of intense high-speed outflows in quasars, and explicitly modeled the interaction between the outflow and host galaxy without using any free parameters and obtained an M- $\sigma$ relation with $A=$ 1.5 and $\alpha=4$. More recent studies on black hole masses in nearby galaxies give $A=1.9$ and $\alpha=5.1$. The tight nature of the $M-\sigma$ relation suggests that a feedback mechanism is operating between the growth of supermassive black holes and the growth of galaxy bulges.

\section{Cosmological Processes in Black Hole Formation}

In this section we explore some basic processes that will set the stage for black hole formation. Accretion around a black hole is one of the key ingredients behind its growth. The second essential element is the existence of a dark matter halo that forms and grows from primordial density fluctuations characterized by a virial radius, a mass overdensity, and a virial temperature. This halo serves as the host for the pre-galactic disk, which usually grows via gas dynamic processes.

\subsection{Accretion around Supermassive Black Holes}

Accretion around supermassive black holes is currently considered the only mechanism capable of producing the observed luminosities produced by supermassive black holes in quasars [29], with a maximum efficiency of $6 \%$ for a non-rotating black hole, and $29 \%$ for a maximally rotating one. Therefore, matter accretion and the subsequent fall on a central supermassive black hole is the main source of the tremendous amount of energy released by active galactic nuclei (AGN). If accretion is an acceptable mechanism for black hole growth, the progenitor remains controversial, with primordial black holes [30], dark stars [31], and collapsing clouds of gas [32] being the main candidates. Growth by the merger of stellar-mass black holes was also evoked as a phase in the formation of supermassive black holes [33]. Cold dark matter, initially at rest, falling freely and radially onto a central black hole would accrete without energy release or observational effect [34]. However, an AGN exhibits a significant amount of angular momentum precludes free infall. The angular momentum of the accreted material that is approaching a central black hole requires a considerable loss of its angular momentum, with a typical loss for a normal galaxy may of about $6 \times 10^{28} \mathrm{~cm}^{2} / \mathrm{s}$ [35] The contribution to such loss of angular momentum may emanate from viscosity, non-axisymmetric gravitational forces, magnetic forces, etc. [35]. For a spherically symmetric potential, the orbit of minimum energy for a fixed angular momentum is a circle, and infalling accreted material resulting from the loss of angular momentum will take the form of successively smaller and smaller concentric circles. Matter describing orbits inclined to each other will eventually collide and there will be a transfer and mixing of angular momenta of different gas streams leading to their equalization. As a consequence, accreted matter tends to orbit in a simple plane having a specific angular momentum at a given radius [35].

The existence of an efficient mechanism for transporting angular momentum outward will enable the accretion material to approach a marginally stable orbit. The existence of a magnetic field in the matter flowing into the disk, as well as turbulent motions, is one such mechanism since it leads to the transfer of angular momentum outward [36]. In accretion disks, particles lose their angular momentum due to friction between adjacent layers, and spiral toward the black hole by releasing their gravitational energy. A certain amount of this energy enhances the kinetic energy of rotation, and the rest is turned into thermal energy irradiated from the disk surface [34].

The energy released in the accretion process and its spectrum are primarily determined by the rate of matter inflow onto the outer boundary of the accretion disk, which converts matter to radiation with an efficiency $\eta$. The Eddington accretion rate is a characteristic scale for accretion, and is given by [34-35]

$$
\dot{M}_{E}=3 \times 10^{-8} \frac{0.06}{\eta} \frac{M}{M_{\odot}}
$$

The total energy released in the disk is equal to the Eddington luminosity

$$
L_{E}=\eta \dot{M} c^{2}
$$

which is a critical luminosity for any given mass $M$, beyond which the radiation force overcomes gravity. Combining the above two equations, we obtain for the Eddington luminosity

$$
L_{E}=1.51 \times 10^{38} \frac{M}{M_{\odot}}
$$

Luminosities ranging from $10^{42}$ to $10^{48} \mathrm{erg} / \mathrm{s}$ have been 
observed for AGNs, corresponding to black hole masses ranging from $10^{5}$ to $10^{9}$ solar masses [35]. Assuming an accretion rate in the AGN of about $0.5 M_{\odot} / \mathrm{yr}$, and a radiation efficiency $\eta \sim 0.1$ gives a resulting luminosity of $3 \times 10^{45} \mathrm{erg} / \mathrm{s}$. This luminosity corresponds to the middle of the observed luminosity distribution of low redshift AGN [35]. The accretion rate of the matter inflow in the accretion disk can assume a wide range of values that may exceed or fall short of the critical Eddington rate. For subcritical accretion $\left(\dot{M} \ll \dot{M}_{E}\right)$, temperatures in the inner region of the disk are of the order of $10^{5}-10^{6} \mathrm{~K}$, corresponding to an energy release in the UV and soft X-ray band [34]. When the accretion rate equals to or exceeds $M_{E}$, the radiation temperature increases to $10^{7}$ or $10^{8} \mathrm{~K}$, and the accretion disk becomes a strong source of X-ray radiation. For a strongly supercritical regime $\left(\dot{M} \gg \dot{M}_{E}\right)$, the luminosity remains fixed at the Eddington critical limit $L_{E}$, whereas most of the irradiated energy from the accretion disk is in the UV and optical region of the spectrum [34]. In the above analysis, we have shown that a good agreement exists between observation and the theory of spectral distribution of radiation from accretion disks of supermassive black holes. However, these theories are mainly concerned with mass accretion rates and the luminosity of the accretion disk irrespective of the origin of the accreting supermassive black hole.

\subsection{Primordial Dark Matter Halos}

Galaxies are thought to be formed from baryonic matter in dark matter halos born out of small primordial density fluctuations [37]. These halos are characterized by a virial radius $r_{\text {vir }}$ representing a sphere containing a mean mass overdensity $\delta_{\text {vir }}$ [38]. There are three important parameters that can be inferred from these halos. The first is the virial mass $M_{\text {vir }}$ that can be calculated directly from the virial theorem. The second is the circular velocity $V_{c}$ which can be calculated from the relation

$V_{c}=\left(G M_{\text {vir }} / r_{\text {vir }}\right)^{1 / 2}$, and the third is the virial temperature

$$
T_{\mathrm{vir}}=\mu m_{p} V_{c}^{2} / 2 k_{B}
$$

where $m_{p}$ is the proton mass, $\mu$ is the mean molecular weight, and $k_{B}$ is the Boltzmann constant. The gravitational collapse of the baryon component can proceed when the mass of the overdense region reaches the Jeans mass $M_{J}$. At masses in excess of the Jeans mass baryons are captured and are then shock-heated by the subsequent collapse and virialization of dark matter. A necessary condition for star formation is that efficient cooling needs to take place, which allows the baryonic cloud to dissipate its kinetic energy and to continue its collapse and fragmentation process [37]. The cooling process is de- termined by how efficient an object is in dissipating energy. Gas dynamics processes predict that low-mass objects are less efficient in dissipating energy and cool rather slowly, whereas more massive objects can cool at a faster rate [39]. The collapsing halos in the early universe exhibit a virial temperature smaller than $10^{4} \mathrm{~K}$, and are referred to as mini halos. At this temperature, cooling is determined by the electron excitation of atomic hydrogen. A necessary condition for the gas to cool down and form the first stars is that the halos should rely on the less efficient $\mathrm{H}_{2}$ cooling [37].

\section{Formation of Supermassive Black Holes}

During the past few decades, several models have been proposed to explain the presence of massive black holes (MBHs) at redshifts corresponding to the era when the universe was less than one billion years old. Important questions to answer are when did the seeding black holes at the centers of galaxies form, and what mechanism was involved in their growth. Several hypotheses have been advanced to elucidate the mechanisms leading to the formation of supermassive black holes. It is pretty much agreed that once a black hole resides at the center of a galaxy, it can grow by accretion or by merging with other black holes. The crucial issue that remains is how the first black holes formed in the first place. The various models we address in this work concern the seed or potential progenitors of supermassive black holes observed at the centers of galaxies. The formation of seeding black holes at high redshifts is an essential requirement of all models. The inferred large masses of massive black holes $(\mathrm{MBH})$ are a core element in all models explaining their origin.

Several possible formation channels have been investigated to understand the $\mathrm{MBH}$ seed, including: 1) the formation and fate of population III stars; 2) the formation mechanism resulting from gas dynamic instabilities, including supermassive stars; 3) formation via stellardynamic processes; 4) collapse of dark stars; 5) primordial black holes from the inflationary era. In the following, we shall introduce the mechanisms suggested by various models leading to the formation of the black holes seed, and compare the predictions of each of these models with the latest observational findings.

\subsection{Collapse of Population III Stars}

Population III stars are massive metal-free objects comprising the first generation of stars after the Big Bang. These stars are postulated to have formed in mini-halos with masses of the order $10^{6} M_{\odot}$ and to have collapsed from the highest primordial density field. For $T_{\text {vir }}>10^{3} \mathrm{~K}$, the cooling process is mediated by molecular hydrogen [39]. Atomic hydrogen cooling takes place in the larger 
halos with a total mass of $10^{8} M_{\odot}$ and $T_{\text {vir }} \geq 10^{4} \mathrm{~K}$. Simulation of the collapse of molecular clouds suggests that massive stars with $M>100 M_{\odot}$ can form [40]. The fate of population III stars depends primarily on their masses. The collapse of $40-140 M_{\odot}$ low-metallicity stars is predicted to directly form a black hole. For this range of masses, the remaining mass is about $40 \%$ of the initial star mass [41]. When the mass of the population III star is in the range of 140 to $260 M_{\odot}$, the fate of the star is determined by the electron-positron pair production instability that leads to supernovae explosions. In these stars, central helium burning drives the core to a temperature and density regime where electron-positron pairs are created in abundance, and can convert the internal energy into rest-mass of the pair with an insignificant contribution to the pressure $[42,43]$. At this stage, depending on the mass of the star, the instability causes a rapid contraction leading either to an implosive oxygen state or to silicon burning. In both cases the collapse is reversed and the star is completely disrupted by a nuclear-power explosion. The core of the star implodes, burns fuel, and explodes violently leaving no remnant $[44,45]$. For yet higher masses $\left(>260 M_{\odot}\right)$, the fate of the star is determined by the photodisintegration instability, which results from the extremely high temperature developed at the center of the star. This process is encountered before explosive burning reverses the implosion [43]. The energy produced in the previous burning stage is rapidly consumed and the collapse continues its momentum to form a black hole $[43,46]$. The final mass of the born black hole may reach at least half the initial stellar mass [45].

Supernovae predicted by this model for certain ranges of massive stars will release a colossal amount of energy that can be detected by current observatories. No such events have been recorded so far. On the other hand, the above model has large uncertainties concerning the final mass of the population III stars. Among these uncertainties is whether a single star or multiple stars are formed per halo. In fact, the initial mass function itself is not well known. For this issue, and other related matters, the reader may consult the following references [47-49].

\subsection{Gas-Dynamic Instabilities}

Metal-free or metal-poor proto-galaxies are efficient nurseries where black holes can be formed and grow. In these systems, supermassive black holes can also be formed directly out of a dense gas cloud [50-52]. On the other hand, enriched halos exhibit an efficient cooling process which favors fragmentation and star formation rather than direct black hole formation. In metal-free gas clouds that characterize the very first proto-galaxies, the collapse is expected to occur only in massive halos with virial temperatures $T_{\text {vir }}>10^{4} \mathrm{~K}$, where the formation of molecular hydrogen is inhibited [53]. At these temperatures $\mathrm{H}_{2}$ formation is inhibited and atomic hydrogen becomes an efficient agent for cooling down the tenuous gas until it reaches $4000 \mathrm{~K}$ [37]. The same process is encountered for slightly enriched gas below the threshold of fragmentation. Suppression of $\mathrm{H}_{2}$ formation requires critical UV fluxes that are much more important than the cosmic UV background. Fragmentation occurs when the gas is enriched above a critical metallicity [54]. At $T_{\text {vir }}>10^{4} \mathrm{~K}$, the line-trapping of Lyman- $\alpha$ photons in isothermally collapsing gas causes the equation of state to stiffen with the consequence that fragmentation becomes harder to achieve provided that the metallicity does not exceed about $10^{-4}$ of the solar metallicity [54]. The dissociation of $\mathrm{H}_{2}$ in these systems is brought about by Lyman- $\alpha$ trapping. In such halos, gas cooling and contraction proceed gradually with no fragmentation until rotational support halts the collapse, which usually occurs before reaching densities that allow the formation of a massive black hole (MBH). Analysis of the rotational dynamics of the collapsing gas shows that tidally induced angular momentum can provide centrifugal support that halts the collapse only at a distance of about $20 \mathrm{pc}$ [37], and ultimately leads to the formation of a disk. Formation of an MBH requires the additional transfer of angular momentum to foster the gas collapse process. Many models have been proposed to provide the additional transfer of angular momentum, leading to the formation of MBH. An efficient mechanism to achieve this transfer was proposed by Shlosman et al. [55] and Begelman et al. [56]. In these models, efficient angular momentum transfer is achieved by dynamical instabilities. These authors proposed the so called "bars-within-bar" mechanism, which originates from the global gravitational instability and dynamical infall. Thus, self-gravitating gas clouds become bar-unstable when the level of rotational support exceeds a certain threshold. A bar is a channel for the outward transportation of angular momentum via gravitational and hydrodynamical torques, which will cause the radius to shrink. Gas cooling and gas shrinking further enhance the instability and cause the process to cascade. This mechanism is successful in accumulating gas at the center of the halos.

Local, rather than global, instabilities in a self-gravitating galactic disk can be calculated using the Toomre stability parameter formalism. The Toomre parameter $Q$ is defined as

$$
Q=\frac{c_{s} \kappa}{\pi G \Sigma}
$$

where $\Sigma$ is the surface mass density, $c_{s}$ is the speed of sound, and $\kappa=\sqrt{2} V / R$ is the epicyclical frequency, and $V$ is the circular velocity of the disk. Gravitational 
instabilities occur when $Q$ approaches a critical value $Q_{c}$. Instabilities might lead to mass infall rather than fragmentation and star formation, provided that destabilization of the system is kept below a threshold value. This happens when the inflow rate is below a critical threshold

$$
\dot{M}_{\max }=2 \alpha_{c} c_{s}^{3} / G
$$

where $\alpha_{c}$ is the viscosity parameter. This process continues until the mass accumulated at the center $\left(M_{a}\right)$ is enough to make the disk marginally stable. The mass is computed from the Toomre instability criteria by requiring that $Q=Q_{c}$, and assuming a DM halo mass that is determined from $T_{\text {vir }} \propto M_{h}^{2 / 3}$ [57]

$$
M_{a}=f_{d} M_{h}\left[1-\left(\lambda / \lambda_{\max }\right)^{1 / 2}\right]
$$

where $\lambda_{\max }=f_{d} Q_{c} / 8\left(f_{d} / j_{d}\right)\left(T_{\text {vir }} / T_{\text {gas }}\right)^{1 / 2}$. Here, $\lambda_{\text {max }}$ is the maximum halo spin parameter, $f_{d}$ is the gas fraction participating in the infall, $j_{d}$ is the fraction of the halo's angular momentum retained by the collapsing gas. The upper limit of the mass that can contribute to $\mathrm{MBH}$ formation is determined by the mass and spin parameter of the halo.

\subsection{Collapse of Supermassive Stars}

Gas dynamical processes can also lead to the formation of supermassive stars (SMS) that may collapse, under certain conditions, to form a $\mathrm{MBH}$. Gas accumulated at the few parsecs around the center of the halo by processes described in the previous section, can reach $10^{4}$ to $10^{6} M_{\odot}$. For efficient gas accumulation, a SMS

$\left(M \simeq 5 \times 10^{4} M_{\odot}\right)$ may form, which eventually collapses to form a black hole [37]. SMS of a given mass, and supported by radiation pressure, will evolve as an $n=3$ polytrope $[58,59]$. It has been shown that the rotation of SMS in a post-Newtonian approximation cannot halt the collapse, and thus an MBH is likely to form [59]. Shibata and Shapiro [60] considered marginally instable and maximally rotating SMS using general relativity and found that the star will form a Kerr-like black hole containing $90 \%$ of the stellar mass.

In systems where mass accumulation is fast enough, the outer layers of the SMS are not thermally relaxed during much of the lifetime of a main sequence star [61]. These stars exhibit complex structures with a convective core surrounded by a convectively stable envelope containing most of the star's mass. Hydrogen burning in the core of these stars is relatively low, and continues throughout most of its massive stages. When hydrogen is exhausted, the SMS will contract and suffer catastrophic neutrino losses that lead to its collapse to an initial black hole with a mass of a few $M_{\odot}$, that grows subsequently via accretion from the resulting bloated envelope. This object is referred to as quasistar [56,62], and it consists of a low-mass central black hole surrounded by a massive radiation-pressure-supported envelope. The black hole grows gradually at the expense of the massive envelope until the resulting $\mathrm{MBH}$ is unveiled. The rate of mass transfer supplied to the black hole's sphere of influence $\left(\dot{M}_{\text {sup }}\right)$ is determined by the Bondi rate evaluated at the black hole's radius of influence. This process is usually suppressed due to the back reaction of the energy flux inside the radius of influence [63]. The accretion rate is thus reduced to $[64,65]$

$$
\dot{M}_{B H} \sim \varepsilon^{-1}\left(c_{s} / c\right)^{2} M_{\text {sup }}
$$

The above relation assumes an absence of a wind that modifies the energy and/or momentum. The quasistar expands gradually and the black hole accretion rate is such that the feedback energy flux equals the Eddington limit. If the feedback average flux exceeds the Eddington limit, the black hole grows at a super-Eddington rate and the photospheric temperature decreases until it reaches a minimum value of about $4 \times 10^{3} \mathrm{~K}$, below which no hydrostatic solution for the convective envelope exists. At this point, the convective zone releases energy at a super-Eddington rate and the final limit of the black hole seed mass is set. The range of masses of the seed is $10^{4}$ to $10^{5} M_{\odot}$, depending on the model.

\subsection{Dynamical Processes in Enriched Halos}

Star formation can proceed in mini-halos characterized by a virial temperature $T_{\text {vir }}<10^{4} \mathrm{~K}[40,66]$. The halos will be enriched with metals by the first generation of population III stars, and thus fragmentation and formation of low mass stars will be a natural outcome of this enrichment [67]. This process sets the stage for new horizons of $\mathrm{MBH}$ formation. Stellar dynamical processes may lead to the formation of compact star clusters [29, $68]$, resulting from collisions. These collisions arise from dynamical interactions and may play a major role in the formation of very massive stars (VMS) leading to the formation of $\mathrm{MBH}$ remnants in the range $10^{2}-10^{4} M_{\odot}$ [69]. In an attempt to reach equilibrium, the compact core cluster initially contracts and then starts to decouple thermally from its outer region. Energy transfer from the central dense core will cause a rapid core collapse [70]. Dynamical friction causes a segregation of more massive stars in the center. If these massive stars remain in the main sequence stage, then a subsystem will be developed and will decouple from the cluster. In this subsystem, star collisions can proceed in a runaway manner eventually leading to the growth of VMSs [71]. The fate of VMSs depends essentially on their metal enrichment. Metal enriched VMSs will lose much of their mass and end their life as less massive objects $\left(\sim 150 M_{\odot}\right)$ [72]. The final fate is either a low-mass black hole or a pair-insta- 
bility supernova. For low metallicity, VMSs may have a different fate. Stars with masses $\geq 40 M_{\odot}$ and sub-solar metallicity may collapse directly into a black hole without a supernova explosion. At a certain level of metallicity, stars start forming in the entire proto-galactic disk. A fraction of about 0.05 of proto-galaxies at $z \sim 10-20$, form black hole seeds of masses $\sim 1000$ - $2000 M_{\odot}$ [69].

\subsection{Dark Stars in the Early Universe}

Dark stars are a new line of research that proposes that the first stars in the universe were fueled by dark matter heating rather than by nuclear fusion [73]. Weakly interacting massive particles (WIMPs) are considered among the best dark matter candidates [74]. It is assumed that in the early universe the density of dark matter was sufficiently high to trigger dark matter annihilation [75]. The annihilation products of WIMPs inside a star can be trapped to produce enough energy to heat its core and prevent its collapse. The first stars are postulated to form inside dark matter halos of masses of the order of $10^{6} M_{\odot}$ [76], with one single star per halo. It is also argued that these stars set the stage for many important processes like reionization, the seeding of supermassive black holes, and the production of heavy elements in subsequent generations of stars. The lightest neutralino is motivated by supersymmetry (SUSY) arguments and is considered the best WIMP candidate in the Minimal Supersymmetric Standard Model [77]. The rate of energy production per unit volume resulting from WIMP annihilation is [76]

$$
Q_{a n n}=\langle\sigma v\rangle_{a n n} \rho_{x}^{2} / m_{x}
$$

where $\langle\sigma v\rangle_{\text {ann }}=3 \times 10^{-26} \mathrm{~cm}^{3} / \mathrm{sec}$ is the annihilation cross-section of weak interaction, and $m_{x}=100 \mathrm{GeV}$ is the WIMP mass. Three key criteria were postulated for dark stars, namely: 1) high dark matter densities; 2) a clumping of annihilation products inside the star; and 3) DM heating overcoming other heating mechanisms. The first criterion is revealed from the above equation, where DM annihilation rate scales as the WIMP density squared $\left(\rho_{x}^{2}\right)$. Dark matter densities in the early universe are assumed to be higher by a factor of $(1+z)^{3}$ [76]. As the protostar forms at the center of the halo, further enhancement occurs resulting from the deepening of the potential well at the center [73]. The second criterion assumes a substantial fraction $f_{Q}$ of the annihilation energy is dissipated in the gas and causes its heating up at a rate of $f_{Q} Q_{a n n}$ per unit volume. Electrons and positrons can deposit energy in the core, whereas neutrinos escape far from the cloud. For the third criterion, it is assumed that a critical transition takes place when the gas density reaches $n>10^{13} \mathrm{~cm}^{-3}$. Above this density, DM heating dominates over all relevant cooling mechanisms, particularly $\mathrm{H}_{2}$ cooling [78]. The first stars that formed this way have $M_{D S}=800 M_{\odot}[76]$. When DM annihilation inside the dark star fades out, it contracts until the temperature reaches $10^{8} \mathrm{~K}$ and fusion sets in. A possible end result is the formation of a supermassive black hole, such as those that have been found at high redshifts, $z=6$, with a mass of $10^{9} M_{\odot}$.

In the dark star model, authors assumed a mass of 100 $\mathrm{GeV}$ for the annihilating WIMPS. So far, WIMPs in general and neutralinos in particular have not been detected despite intensive searches during the past few decades. Furthermore, no trace of supersymmetric particles has been found in the Large Hadron Collider (LHC), even though it attains energies of seven tera-electron volts, which is far in excess of the $100 \mathrm{GeV}$ postulated for annihilating DM particles in dark stars. Also, it is assumed in the dark star model that annihilating particles are the source of heat or thermal radiation. All observations so far confirm that DM interacts with normal matter only gravitationally, resulting in lensing effects of faraway background galaxies. Theoretical work [79] suggests that the interaction of DM with baryonic matter is of a gravitational nature only with no electromagnetic component. A recent review of DM [80] indicates that its origin may defy conventional ideas and belongs to the realm of extra-dimensions postulated by superstring theories. In short, the DM model in its present form is incapable of addressing some fundamental issues about the origin of DM and its presumed interaction with baryonic matter.

\subsection{Primordial Black Holes}

Theoretical studies of the possibility of formation of primordial black holes $(\mathrm{PBH})$ in the early universe date back to the original work of Hawking [1]. He argued that extreme densities and inhomogenities in the early universe can lead to the local collapse of matter resulting in the formation of black holes. More recently, Choptuik [81] and Kim [82] demonstrated the formation of PBHs in the inflationary era, during which the energy density of the universe experienced a dramatic decrease leading to a cosmological phase transition. Hawking [1] argues that PBHs formed in a wide spectrum of masses in the early universe ranging from $10^{-5} \mathrm{~g}$, corresponding to the Planck mass, to $10^{17}$ solar masses. His upper limit for mass exceeds, by many orders of magnitude, even the greatest masses of supermassive black holes observed today in galactic centers. On the other hand, the formation of very small black holes may arise either from the softening of the equation of state [83], phase transitions [84], or from the collapse of hypothetical cosmic strings [85].

Overdense regions in the early universe may collapse to a black hole if the gravitational attraction overcomes 
the pressure forces and the velocity of expansion [74]. This condition is fulfilled when the potential energy for self-gravitation

$$
\Omega \sim-\mu^{2} R^{5}
$$

exceeds the kinetic energy of expansion

$$
T \sim-\mu R^{3} \dot{R}^{2}
$$

where $R$ is the radius of a region in the early universe, and $\mu$ is the energy density. The units are such that $G=c=1$. In a $k=0$ Friedman universe the sum of these energies is zero. Therefore

$$
\left(\frac{\dot{R}}{R}\right)^{2} \sim \mu
$$

Furthermore, Hawking assumed that the equation of state relating the pressure $P$ and the energy density $\mu$ has the form $P=\mu / 3$, and that $\mu$ is proportional to $R^{-4}$. Thus,

$$
\mu \sim t^{-2}, \text { and } R \propto \sim t^{-1 / 2}
$$

A necessary condition for the collapse to occur is that the gravitational energy, $\mu$, should exceed the internal energy $U$. Taking $P=\mu / 3$ and $U \sim \mu \sim R^{3}$, the condition for collapse to occur becomes

$$
\mu R^{2}>1
$$

for $P \sim \mu_{\tilde{o}} \log \mu / \mu_{o}$, and $U \sim \mu_{o} R^{3} \log \mu / \mu_{o}$, the condition for collapse reduces to

$$
\mu R^{2}>\frac{\mu}{\mu_{o}} \log \frac{\mu}{\mu_{o}}
$$

Once a black hole is formed, it will grow by accreting nearby matter. The rate of accretion was calculated by Zeldovitch and Navikov [86]

$$
\frac{\mathrm{d} M}{\mathrm{~d} t} \sim \mu \sim R_{g}^{2} \sim \mu \sim M^{2}
$$

where $\mu$ here is the density of the background universe. But since $\mu=t^{-2}$ (see above), hence

$$
M \sim \frac{t}{1+\frac{t}{t_{o}}\left(\frac{t_{o}}{M_{o}}-1\right)}
$$

where $M_{o}$ is the initial mass of the black hole and $t_{o}$ is the time of formation. Thus, if $M_{o}$ is small compared to $t_{o}$, that is, if the black hole is small compared to the particle horizon, then $M-M_{o}$ remains small and there would be almost no accretion. However, if $M_{o}$ is of the same order as $t_{o}$, then the Zeldovitch-Navikov argument leads to $M \sim t_{o}$. In this case, the accretion would cause the black hole to grow at the same rate as the particle horizon, producing black holes of the order of the
Hubble radius if the growth continued to the present time, or it would reach a mass of $10^{15}$ to $10^{17}$ solar masses if the growth was at the same rate as that of the particle horizon.

Carr and Rees [85] demonstrated that the density fluctuations of a Gaussian distribution lead to a probability that a given region evolves into a black hole given by

$$
P \approx \varepsilon \exp \left(-\alpha^{2} / 2 \varepsilon^{2}\right)
$$

where $\alpha$ is a constant defined by the equation of state $P=\alpha \mu$, and $\varepsilon$ is a constant. A black hole is unlikely to form for $\alpha$ of the order of 1 and for $\varepsilon$ much less than 0.1. Lin et al. [86] demonstrated that the Einstein equation for a stiff equation of state $(P=\mu)$ permits a spherically symmetric solution in which pressure gradients cause a black hole to grow as fast as the universe. It was argued that only hot models of the early universe are capable of producing PBHs prolifically enough to be consistent with observations. Harada [87] considered the growth of super-horizon PBHs, assuming a mass scale $M_{h f} \simeq \sim G^{-1} c^{3} t_{f}$ which is contained in the Hubble horizon of the formation epoch. Here, $G, c$ and $t_{f}$ are, respectively, the gravitational constant, the speed of light, and the formation time from the Big Bang. A typical mass scale of PBHs corresponds to the horizon mass scale of the formation epoch

$$
M_{P B H, f}=M_{h, f}=\frac{c^{3} t_{f}}{G} \approx M_{\odot}\left(\frac{T_{f}}{100 \mathrm{MeV}}\right)^{-2}
$$

and the mass accretion rate for a black hole was estimated as

$$
\frac{\mathrm{d} M}{\mathrm{~d} t}=4 \pi \alpha r_{A} v_{s} \rho_{\infty}
$$

where $r_{A}=G M / v_{s}^{2}$ is the accretion radius, $v_{s}$ is the speed of sound, $\rho_{\infty}$ is the density at infinity, and $\alpha$ is a constant of order unity. Harada [87] assumed that $\rho_{\infty}$ is given by the density of the background Friedman universe, and $v_{s}$ is of the order of the speed of light. In this case, the accretion rate can be integrated to give

$$
M=\frac{A t}{1+\frac{t}{t_{f}}\left(\frac{A t_{f}}{M_{f}}+1\right)}
$$

where $A \approx \sim c^{3} / G$ is a constant, and $M_{f}$ is the mass of the $\mathrm{PBH}$ at the formation time $\left(t_{f}\right)$ relative to the Big Bang. Harada [87] obtained three categories of solutions, namely: sub-horizon, self-similar, and super-similar. In his paper, the effect of cosmological expansion was neglected, since it is important only for the cosmological horizon scale. The above analysis is expected to be valid only for PBHs much smaller than the cosmological horizon scale. 


\subsection{Hawking Evaporation of Black Holes}

Quantum gravitation effects are usually neglected when calculating the formation and evaporation of black holes. The justification for this approach is that the radius of curvature of spacetime outside the event horizon is very large compared to the Planck length

$\left(G \hbar / c^{3}\right)^{1 / 2} \approx 10^{-33} \mathrm{~cm}$, which is the length scale on

which quantum fluctuations of the metric are expected to be of the order of unity [88]. Hawking derived an expression for the emission spectrum that mimics a Planck radiation law using the usual quantum mechanical wave equation for a collapsing object with a post collapse classical wave metric. He showed that quantum mechanical effects cause black holes to create and emit particles as if they were blackbodies of temperature

$$
T=\frac{h c^{3}}{16 \pi k G M} \approx \sim 10^{-16}\left(\frac{M_{\odot}}{M}\right) \mathrm{K}
$$

Thus, the Hawking temperature is inversely proportional to the black hole mass $M$. Therefore, as the black hole radiates, its temperature increases. The evaporation of black holes was a source of controversy for some time. To solve this dilemma, detection of PBHs was the subject of intense research since their existence was postulated in the early 1970s. Hawking [88] and Page [89] have shown that in order for PBH evaporation to occur in our current epoch, they must have a mass $M \leq 10^{15} \mathrm{~g}$. The evaporation is accompanied by a burst of high energy particles and gamma rays [90]. The clustering of PBHs was considered by Page and Hawking [90]. They found that for $M \approx 10^{15} \mathrm{~g}$, the maximum allowed space density of PBHs is $n(M) \leq 10^{11} \mathrm{Cpc}^{-3}$, where $C$ is the clumping factor. Cline [91] relied on data obtained from the EGRET detector aboard the Compton Gamma Ray Observatory to attribute some of the observed gammaray flux to the evaporation of PBHs, which is strongly clustered in the galactic halo. Furthermore, he argued that the galactic gamma-ray halo arises primarily from the evaporation of PBHs. Accepting the data at face value implies that the existence of PBHs no longer resides in the realm of theoretical speculation. Several constraints limit the mass range of primordial black holes that can be observed. As mentioned above, black holes with an initial mass smaller than $M \approx 10^{15} \mathrm{~g}$ are expected to be already evaporated. A constraint is set for more massive black holes as determined by microlensing techniques [92] and from spectral distortions of the cosmic background radiation [93].

\section{The Connection between Black Holes and Gamma-Ray Bursts}

Gamma-ray bursts (GRBs) are the most powerful explo- sions in the universe, and hold great promise as cosmological probes of the early universe. They were serendipitously discovered in the late 1960s [94], and although a great deal of effort has gone into understanding these enigmatic explosions, the precise physical mechanism behind their formation remains elusive. Traditionally, GRBs have been classified, based on duration, into long bursts (LGRBs) with $T_{90}>2 \mathrm{~s}$, and short bursts (SGRBs) with $T_{90}<2 \mathrm{~s}$, where $T_{90}$ is the duration needed to accumulate $90 \%$ of the burst's fluence [95]. However, some recent studies have provided evidence for a third class of GRBs called Very Short Gamma-Ray Bursts (VSGRBs) with a $T_{90}<0.1 \mathrm{~s}$ [96]. For some recent reviews on GRBs, the reader is referred to [97-102]. In this section, we will explore the connection between black holes and all three classes of GRBs.

According to current theoretical models, the formation of GRBs is intimately related to black holes regardless of whether we are dealing with long, short, or even very short bursts. We shall start off by investigating this connection for LGRBs and SGRBs, and then consider VSGRBs where the mechanism is somewhat different.

The leading progenitor model for the formation of LGRBs is the so-called collapsor model. In this model, the rotating core of a massive star collapses, and the released energy is channeled out in the form of relativistic beams $[103,104]$. According to this model, the minimum angular momentum needed is basically the value associated with the last stable orbit around a black hole which, for a non-rotating black hole, is given by [102]:

$$
J=2(3)^{1 / 2} G M / c=4.6 \times 10^{16}\left[M_{B H} / 3 M_{\odot}\right] \mathrm{cm}^{2} / \mathrm{s}
$$

and for a rotating black hole with a Kerr parameter $a=1$, it is given by [9]:

$$
J=2 G M /(3)^{1 / 2} c=1.5 \times 10^{16}\left[M_{B H} / 3 M_{\odot}\right] \mathrm{cm}^{2} / \mathrm{s}
$$

where $G$ is the universal gravitational constant, $c$ is the speed of light in vacuum, $M_{B H}$ is the mass of the black hole, and $M_{\odot}$ is the mass of the sun.

Two conditions that should be kept in mind concerning the collapsor model are that in order for this model to work the jets must be able to pierce through the star and escape, and the star should not explode prematurely, otherwise a black hole will not form [102]. Recent investigations indicate that the Blandford-Znajek mechanism [105] is probably behind the formation of the relativistic jets, and these energy-loaded jets, when injected near the center of a massive star, are able to penetrate the star and form a streaming jet with the necessary opening angle and Lorentz factor [102].

The progenitors of SGRBs are currently believed to be mergers of compact stars: two neutron stars or a neutron star and a black hole. Either scenario is expected to lead 
to the formation of a black hole that is surrounded by a torus of debris [106]. Since there is no external feeding of the accretion disk, then the event is not expected to last too long - on the order of one second. The two main sources of energy that act as a reservoir for the SGRB are the binding energy of the material that is orbiting in the disk, and the black hole's spin energy. In fact, it has been estimated that up to $29 \%$ of the black hole's rest-mass energy and about $42 \%$ of the rest-mass found in the disk can be extracted and utilized to power the SGRBs [106]. If $\mathrm{d} M / \mathrm{d} t$ is the mass inflow rate, then to support the SGRB, it must be at least [106]:

$$
\mathrm{d} M / \mathrm{d} t=3 \times 10^{-3}(0.1 / e)\left[L_{S G R B} / 10^{51} \mathrm{erg} \cdot \mathrm{s}^{-1}\right] M_{\odot} \cdot \mathrm{s}^{-1}
$$

where $L_{S G R B}$ is the luminosity of the SGRB and $\varepsilon$ is the overall efficiency.

Black holes are also intimately involved in the production of VSGRBs. According to [96], VSGRBs constitute a separate class of GRBs for several observational reasons. First, VSGRBs show considerable anisotropy in their galactic angular distribution, and their $V / V_{\max }$ distribution also points to a "local" distance scale. Furthermore, only a small percentage (about 25\%) have observed afterglows, compared to about $78 \%$ for SGRBs.

VSGRBs can be produced by primordial black holes (PBHs). A supporting piece of evidence is that the rising part of the time profiles of VSGRBs is in good agreement with $\mathrm{PBH}$ evaporation models [3]. The extremely high temperatures and pressures that existed right after the Big Bang were conducive to the production of PBHs. Fluctuations in the density of matter could have produced PBHs that persisted to our present time [107-109]. According to [107], PBHs with masses less than about $5 \times$ $10^{11} \mathrm{~kg}$ would have evaporated by now. These evaporating PBHs can be detected through their Hawking radiation, and may appear as very short bursts as modeled in [110], with time durations of the order of $0.1 \mathrm{~s}$ and with a luminosity of about $10^{33} \mathrm{erg} / \mathrm{s}$.

From what has been stated in this section, it seems reasonable to conclude that black holes are intimately involved in the production of all three classes of GRBs: long, short, and very short bursts.

\section{Conclusion}

In this paper, the various scenarios for the formation of seed black holes in the early universe and their subsequent growth to supermassive black holes were presented. The M- $\sigma$ relation suggests a common root for black holes and active galactic nuclei. Cosmological processes involving the seeding primordial dark halos and the pregalactic accretion disks were introduced in order to shed light on the subsequent paths for the formation of galaxies. The various channels leading to the formation of supermassive black holes were discussed as well. Metallic enrichment and virial temperatures were found to play a major role in determining the behavior of gas dynamics in pre-galactic discs, and thus in the subsequent formation of the seeding black holes. Metal-free or metal-poor environments favor the formation of population III stars that end their lives as massive black holes. In this environment, black holes can also be formed directly out of a dense gas cloud. In metal rich environments, fragmentation and star formation is the dominant mechanism. Black holes are formed in these systems by the collision of stars in clusters and the subsequent sinking to the center of the pre-galactic disk. Gas dynamical processes can also lead to the formation of supermassive stars that end their lives as massive black holes. Dark stars, as a possible channel leading to the formation of massive black holes, were also discussed. No observational evidence has been found so far for the existence of supersymmetric particles, which are presumed to be responsible for producing the necessary heat at the core of dark stars, despite the fact that energies well above that postulated for their existence have been probed. The formation of primordial black holes from the inflationary era was also reviewed. Theoretical studies show that these objects were formed with a wide spectrum of masses. However, it should be kept in mind that distortion signatures in the microwave background radiation set a limit on the masses of primordial black holes. Hawking evaporation is a key element in exploring the validity of primordial black hole models. Recent observations are in favor of their existence. Furthermore, we discussed the important connection between the powerful stellar explosions known as gamma-ray bursts and black holes, and how understanding one of them may lead to a better understanding of the other.

\section{REFERENCES}

[1] S. W. Hawking, Monthly Notices of the Royal Astronomical Society, Vol. 152, 1971, p. 75.

[2] S. W. Hawking, Nature, Vol. 248, 1974, pp. 30-31. doi:10.1038/248030a0

[3] B. J. Carr, Astrophysical Journal, Vol. 205, 1975, p. 1. doi: $10.1086 / 153853$

[4] J. Silk and M. J. Rees, Astronomy \& Astrophysics, Vol. 331, 1998, pp. L1-L4.

[5] R. Barakana and A. Loeb, Physics Reports, Vol. 349, 2001, pp. 125-238. doi:10.1016/S0370-1573(01)00019-9

[6] M. Volonteri, F. Haardt and Madau, Astrophysical Journal, Vol. 582, 2003, pp. 559-573. doi:10.1086/344675

[7] S. Tremaine, et al., Astrophysical Journal, Vol. 574, 2002, pp. 740-753.

[8] A. J. Barth, et al., Astrophysical Journal Letters, Vol. 594, 2003, pp. L95-L98. 
[9] C. J. Willott, et al., Astronomical Journal, Vol. 134, 2007, pp. 2435-2450. doi:10.1086/522962

[10] Z. Haimian, Astronomical Journal, Vol. 613, 2004, pp. 36-40.

[11] A. Loeb and F. A. Rasio, Astrophysical Journal, Vol. 432, 1994, pp. 52-61. doi:10.1086/174548

[12] X. Fan, et al., Astronomical Journal, Vol. 121, 2001, pp. 54-65. doi:10.1086/318033

[13] A. M. Ghez, et al., Astronomical Journal, Vol. 620, 2005, pp. 744-754.

[14] R. Bender, et al., Astronomical Journal, Vol. 631, 2005, pp. 280-300. doi:10.1086/432434

[15] F. Macchetto, et al., Astronomical Journal, Vol. 489, 1997, p. 579.

[16] K. Gebhardt and J. Thomas, Astronomical Journal, Vol. 700, 2009, pp. 1690-1701. doi:10.1088/0004-637X/700/2/1690

[17] N. J. McConnell, Nature, Vol. 480, 2011, pp. 215-218. doi:10.1038/nature10636

[18] M. J. Valtonen, et al., Nature, Vol. 452, 2008, pp. 851853. doi:10.1038/nature06896

[19] E. Treister, et al., Nature, Vol. 474, 2011, pp. 356-358. doi: 10.1038/nature10103. doi:10.1038/nature10103

[20] L. L. Cowie, A. J. Barger and G. Hasinger, Astronomical Journal, Vol. 748, 2012, p. 50. doi: $10.1088 / 0004-637 \mathrm{X} / 748 / 1 / 50$

[21] C. Willott, Astronomical Journal, Vol. 742, 2011, pp. L8L11. doi:10.1088/2041-8205/742/1/L8

[22] D. Merritt, "Dynamics and Evolution of Galactic Nuclei," Princeton University Press, Princeton, 2013.

[23] B. M. Peterson and K. Horne, "Reverberation on Mapping of Active Galactic Nuclei," In: M. Livio and S. Casertano, Planets to Cosmology: Essential Science in the Final Years of the Hubble Space Telescope, Proceedings of the Space Telescope Science Institute Symposium, Baltimore, 3-6 May 2004, Cambridge University Press, Cambridge, Space Telescope Science Institute Symposium Series, Vol. 18, 2006.

[24] D. Merritt, "Black Holes and Galaxy Evolution," In F. Combes, G. A., Mamon and V. Charmandaris, Dynamics of Galaxies: From the Early Universe to the Present, Astronomical Society of the Pacific, 1999, pp. 221-232.

[25] F. Ferrarese and D. Merritt, Astronomical Journal, Vol. 539, 2000, pp. L9-L12. doi:10.1086/312838

[26] K. Gebhardt et al., Astronomical Journal, Vol. 539, 2000, pp. L13-L16.

[27] D. Merritt and F. Ferrarese, Astronomical Journal, Vol. 547, 2001, pp. 140-145. doi:10.1086/318372

[28] A. King, Astronomical Journal, Vol. 596, 2003, pp. L27L29. doi:10.1086/379143

[29] P. Schneider, "Extragalactic Astronomy and Cosmology: An Introduction," Springer-Verlag, Heidenberg, 2006.

[30] A. Barrau, Astroparticle Physics, Vol. 12, 2000, pp. 269275. doi:10.1016/S0927-6505(99)00103-6

[31] K. Freese, P. Gondolo and D. Spolyar, "The Effect of
Dark Matter on the First Stars: A New Phase of Stellar Evolution," Proceedings of First Stars III, Santa Fe, 1620 July 2008, pp. 42-44.

[32] S. M. Koushiappas, J. S. B. Bullock and A. Dekel, Monthly Notices of the Royal Astronomical Society, Vol. 354, pp. 292-304.

[33] M. C. Miller and V. M. Lauburg, Astrophysical Journal, Vol. 692, 2009, p. 917.

[34] N. I. Shakura and R. A. Sunyaev, Astronomy and Astrophysics, Vol. 24, 1973, pp. 337-355.

[35] J. H. Krolik, “Active Galactic Nuclei," Princeton University Press, Princeton, 1999.

[36] P. Jovanovic and L. C. Popovoc, "X-Ray Emission from Accretion Disks of AGN," In: A. D. Wacher and R. J. Propst, Eds., Black Holes and Galaxy Formation, Nova Science Publishers, Inc., Hauppauge, 2010.

[37] M. Volonteri, The Astronomy and Astrophysics Review, Vol. 18, 2010, pp. 279-315. doi:10.1007/s00159-010-0029-x

[38] V. R. Eke, S. Cole and C. S. Frenk, Monthly Notices of the Royal Astronomical Society, Vol. 282, 1996, pp. 263280. doi:10.1093/mnras/282.1.263

[39] M. Tegmark, et al., The Astrophysical Journal, Vol. 474, 1997, p. 1.

[40] V. Bromm, P. S. Coppi and R. B. Larson, The Astrophysical Journal Letters, Vol. 527, 1999, pp. L5-L8. doi: $10.1086 / 312385$

[41] W. Zhang, S. E. Woosley and A. Heger, The Astrophysical Journal, Vol. 679, 2008, pp. 639-654. doi:10.1086/526404

[42] Z. Barkat, G. Rakavy and N. Sack, Physical Review Letters, Vol. 18, 1967, pp. 379-381.

[43] J. R. Bond, W. D. Arnett and B. J. Carr, The Astrophysical Journal, Vol. 280, 1984, pp. 825-847.

[44] R. P. Kudritzki and J. Puls, Annual Review of Astronomy and Astrophysics, Vol. 38, 2000, pp. 613-666.

[45] C. L. Fryer, S. E. Woosley and A. Heger, The Astrophysical Journal, Vol. 550, 2001, pp. 372-382. doi:10.1086/319719

[46] S. E Woosley and T. A. Weaver, Annual Review of Astronomy and Astrophysics, Vol. 24, pp. 205-253. doi:10.1146/annurev.aa.24.090186.001225

[47] S. C. O. Glover, et al., IAU Symposium, Vol. 255, 2008, pp. 3-17.

[48] M. J. Turk, T. Abel and B. O'Shea, Science, Vol. 325, 2009, pp. 601-605. doi:10.1126/science. 1173540

[49] M. Trenti, et al., The Astrophysical Journal, Vol. 700, 2009, pp. 1672-1679. doi:10.1088/0004-637X/700/2/1672

[50] M. G. Haehnelt and M. J. Rees, Monthly Notices of the Royal Astronomical Society, Vol. 263, 1993, pp.168-178.

[51] A. Loeb and F. A. Rasio, The Astrophysical Journal, Vol. 432, 1994, pp. 52-61. doi:10.1086/174548

[52] G. Lodato and P. Natarajan, Monthly Notices of the Royal Astronomical Society, Vol. 371, 2006, pp. 1813-1823.

[53] V. Bromm and A. Loeb, The Astrophysical Journal, Vol. 
596, 2003, pp. 34-46. doi:10.1086/377529

[54] F. Santoro and J. M. Shull, The Astrophysical Journal, Vol. 643, 2006, pp. 26-37. doi:10.1086/501518

[55] I. Shlosman, J. Frank and M. C. Begelman, Nature, Vol. 338, 1989, pp. 45-47. doi:10.1038/338045a0

[56] M. C. Begelman, M. Volonteri and M. J. Rees, Monthly Notices of the Royal Astronomical Society, Vol. 370, 2006, pp. 289-298.

[57] H. J. Mo, S. Mao and S. D. M. White, Monthly Notices of the Royal Astronomical Society, Vol. 295, 1998, pp. 319336. doi:10.1046/j.1365-8711.1998.01227.x

[58] T. W. Baumgate and S. L. Shapiro, The Astrophysical Journal, Vol. 526, 1999, pp. 941-952. doi:10.1086/308006

[59] M. Saijo, et al., The Astrophysical Journal, Vol. 569, 2002, pp. 349-361. doi:10.1086/339268

[60] M. Shibata and S. L. Shapiro, Astrophysical Journal Letters, Vol. 572, 2002, pp. L39-L43. doi:10.1086/341516

[61] M. C. Begelman, Monthly Notices of the Royal Astronomical Society, Vol. 402, 2009, pp. 673-681.

[62] M. C. Begelman, E. M. Rossi and P. J. Armitage, Monthly Notices of the Royal Astronomical Society, Vol. 387, 2008, pp. 1649-1659.

doi:10.1111/j.1365-2966.2008.13344.x

[63] A. V. Gruzinov, The Astrophysical Journal, Vol. 501, 1998, p. 787. doi:10.1086/305845

[64] R. D. Blandford and M. C. Begelman, Monthly Notices of the Royal Astronomical Society, Vol. 303, 1999, pp. L1L5. doi:10.1046/j.1365-8711.1999.02358.x

[65] R. D. Blandford and M. C. Begelman, Monthly Notices of the Royal Astronomical Society, Vol. 349, 2004, pp. 6886. doi:10.1111/j.1365-2966.2004.07425.x

[66] T. Abel, G. L. Bryan and M. L. Norman, The Astrophysical Journal, Vol. 540, 2000, pp. 39-44. doi:10.1086/309295

[67] K. Omukai, R. Schneider and Z. Haiman, The Astrophysical Journal, Vol. 686, 2008, pp. 801-814. doi:10.1086/591636

[68] P. C. Clark, S. C. O. Glover and R. S. Klessen, The Astrophysical Journal, Vol. 672, 2008, pp. 757-764. doi: $10.1086 / 524187$

[69] B. Devecchi and M. Volonteri, The Astrophysical Journal, Vol. 694, 2009, pp. 302-313. doi:10.1088/0004-637X/694/1/302

[70] L. Spitzer, "Dynamical Evolution of Globular Clusters," Princeton University Press, Princeton, 1987.

[71] Z. S. F. Portegies, et al., Astrophysics, Vol. 348, 1999, pp. 117-126.

[72] E. Gaburov, J. Lombardi and Z. S. Portegies, Astrophysics, Vol. 402, 2009, pp. 105-126.

[73] D. Spolyar, K. Freese and P. Gondolo, Physical Review Letters, Vol. 100, 2008, Article ID: 051101.

[74] J. L. Feng, Annual Review of Astronomy and Astrophysics, Vol. 48, 2010, pp. 495-545. doi:10.1146/annurev-astro-082708-101659
[75] L. Krauss, et al., The Astrophysical Journal, Vol. 299, 1985, pp. 1001-1006. doi:10.1086/163767

[76] K. Freese, et al., "Dark Stars: The First Stars in the Universe may be Powered by Dark Matter Heating," 2008. arXiv:0812.4844v1

[77] J. Primack, D. Seckel and B. Sadoulet, Annual Review of Nuclear and Particle Science, Vol. 38, 1988, pp. 751-807. doi:10.1146/annurev.ns.38.120188.003535

[78] D. Hollenbach and C. F. McKee, Astrophysical Journal Supplement Series, Vol. 41, 1979, pp. 555-592. doi:10.1086/190631

[79] L. S. Schlman, Physical Review Letters, Vol. 83, 1999, pp. 5419-5422. doi:10.1103/PhysRevLett.83.5419

[80] S. Al Dallal and W. J. Azzam, Journal of Modern Physics, Vol. 3, 2012, pp. 1131-1141. doi:10.4236/jmp.2012.329148

[81] M. W. Choptuik, Physical Review Letters, Vol. 70, 1993, pp. 9-12. doi:10.1103/PhysRevLett.70.9

[82] H. I. Kim, Physical Review D, Vol. 62, 2000, Article ID: 063504. doi:10.1103/PhysRevD.62.063504

[83] V. Canuto, Monthly Notices of the Royal Astronomical Society, Vol. 184, 1978, pp. 721-725.

[84] S. W. Hawking, I. G. Moss and J. M. Stewart, Physical Review D, Vol. 26, 1982, pp. 2681-2713. doi:10.1103/PhysRevD.26.2681

[85] S. W. Hawking, Physics Letters B, Vol. 231, 1989, pp. 237-239. doi:10.1016/0370-2693(89)90206-2

[86] Y. Zel'dovich and I. D. Navikov, "Stars and Relativity," University of Chicago Press, Chicago, 1971.

[87] T. Harada, Journal of Physics: Conference Series, Vol. 31, 2006, pp. 111-114.

[88] S. W. Hawking, Communications in Mathematical Physics, Vol. 43, 1975, pp. 199-220. doi:10.1007/BF02345020

[89] D. N. Page, Physical Review D, Vol. 13, 1976, pp. 198206. doi:10.1103/PhysRevD.13.198

[90] D. N. Page and S. W. Hawking, The Astrophysical Journal, Vol. 206, 1976, pp. 1-7. doi:10.1086/154350

[91] D. B. Cline, The Astrophysical Journal, Vol. 501, 1998, pp. L1-L3. doi:10.1086/311433

[92] P. Tisserand, et al., Astrophysics, Vol. 469, 2007, pp. 387-404. doi:10.1051/0004-6361:20066017

[93] M. Ricotti, J. P. Striker and J. K. Mack, The Astrophysical Journal, Vol. 680, 2008, pp. 829-845. doi: $10.1086 / 587831$

[94] R. W. Klebesadel, et al., The Astrophysical Journal, Vol. 182, 1973, pp. L85-L88. doi:10.1086/181225

[95] C. Kouveliotou, et al., The Astrophysical Journal, Vol. 413, 1993, pp. L101-L104. doi:10.1086/186969

[96] D. B. Cline, et al., International Journal of Astronomy and Astrophysics, Vol. 1, 2011, pp. 164-172. doi:10.4236/ijaa.2011.13021

[97] N. Gehrels and P. Mészáros, Science, Vol. 337, 2012, pp. 932-936. doi:10.1126/science. 1216793

[98] B. Zhang, Chinese Journal of Astronomy and Astrophy- 
sics, Vol. 7, 2007, pp. 1-50. doi:10.1088/1009-9271/7/1/01

[99] B. Zhang, Chinese Journal of Astronomy and Astrophysics, Vol. 7, 2007, p. 329. doi:10.1088/1009-9271/7/2/18

[100] N. Gehrels, et al., Annual Review of Astronomy and Astrophysics, Vol. 47, 2009, pp. 567-617. doi:10.1146/annurev.astro.46.060407.145147

[101] G. Vedrenne and J.-L. Atteia, “Gamma-Ray Bursts," Springer, Berlin, 2009. doi:10.1007/978-3-540-39088-6

[102] C. Kouveliotou, et al., "Gamma-Ray Bursts," Cambridge University Press, Cambridge, 2012. doi:10.1017/CBO9780511980336

[103] S. E. Woosley, Astrophysical Journal, Vol. 405, 1993, pp. 273-277.

[104] A. I. MacFadyen and S. E. Woosley, Astrophysical Journal, Vol. 524, 1999, pp. 262-289. doi:10.1086/307790
[105] R. D. Blandford and R. L. Znajek, Monthly Notices of the Royal Astronomical Society, Vol. 179, 1977, pp. 433-456.

[106] W. H. Lee and E. Ramirez-Ruiz, New Journal of Physics, Vol. 9, 2007, p. 17. doi:10.1088/1367-2630/9/1/017

[107] S. W. Hawking, Nature, Vol. 248, 1974, pp. 30-31. doi:10.1038/248030a0

[108] B. J. Carr, et al., Physical Review D, Vol. 81, 2010, Article ID: 104019 . doi:10.1103/PhysRevD.81.104019

[109] J. B. Carr and S. W. Hawking, Monthly Notices of the Royal Astronomical Society, Vol. 168, 1974, pp. 399-416.

[110] D. B. Cline and W. P. Hong, Astrophysical Journal, Vol. 401, 1992, pp. L57-L60. doi:10.1086/186670 